American Journal of Applied Sciences 6 (3): 471-477, 2009

ISSN 1546-9239

(C) 2009 Science Publications

\title{
Ramadan Fasting Diet Entailed a Lipid Metabolic Disorder Among Type 2 Diabetic Obese Women
}

\author{
${ }^{1}$ Méghit Boumédiène Khaled and ${ }^{2}$ Slimane Belbraouet \\ ${ }^{1}$ Department of Biology, Faculty of Sciences, Djillali Liabès University, Sidi-bel-Abbes, Algeria \\ ${ }^{2}$ School of Nutrition, University of Moncton, Moncton, E1A 3E9, Canada
}

\begin{abstract}
We designed this study to assess the effect of diet during Ramadan fasting on body weight and on serum lipid components in type 2 diabetic obese women. During Ramadan of 2005, 89 diabetic women receiving oral treatment $\left(\mathrm{BMI}=33.20 \pm 3.01 \mathrm{~kg} \mathrm{~m}^{-2}\right)$, aged $52( \pm 5$ years $)$, were selected. The study was carried out over 3 periods-, before (T1: pre-fasting), during (T2: fasting), and after (T3: post-fasting) Ramadan-in Sidi-bel-Abbes city. The daily food intake during the course of 3 days was recorded, and then weight, height, waist and hip circumferences, Body Mass Index (BMI) and Waist to Hip Ratio (WHR) were measured. Blood samples were analyzed for total cholesterol (TC), low-density lipoprotein cholesterol (LDL-c), high-density lipoprotein cholesterol (HDL-c), apolipoprotein A1 (apoA-I), apolipoprotein B (apoB), triglycerides (TGs), fasting serum glucose (FSG), and glycated haemoglobin (HbA1c) and results were compared using Student's t-test. The dietary fat consumption increased during Ramadan, especially for the saturated one and as a result induced a major lipid unbalance, marked by a disorder observed in nearly all of the serum lipid components. There was a significant weight loss in diabetic women $(-2.56 \mathrm{~kg}, \mathrm{p}<0.05)$ during $\mathrm{T} 2$, correlated with a decrease in meal frequency $\left(\mathrm{r}^{2}=0.313, \mathrm{p}<0.01\right)$. As well, the level of glucose decreased significantly during $\mathrm{T} 2$ $\left(8.23 \pm 0.75 \mathrm{mmol} \mathrm{L}^{-1}\right)$ compared to $\mathrm{T} 1\left(9.23 \pm 1.08 \mathrm{mmol} \mathrm{L}^{-1}\right)$. Our study showed a significant weight loss during Ramadan period and an improvement of glucose homeostasis. However, the lipid metabolism was imbalanced and was strongly linked to the Ramadan diet and to the eating habits modification.
\end{abstract}

Key words: Ramadan fasting, type 2 diabetes, obesity, lipid parameters, weight loss

\section{INTRODUCTION}

Ramadan fasting, the ninth month of Islamic Hijri Calendar is a religious obligation of Islam, annually followed by millions of Muslims to fulfil their worship and to abstain from food and water from dawn to sunset. Ramadan fasting is commonly seen as beneficial for health. Among disabled individuals with acute or chronic diseases, certain diabetics can be exempted from this sacred obligation. On one hand, many people with this long-term -illness still prefer to observe fasting, without medical guidance, exposing themselves to certain health risks as a direct consequence of fasting, or of a change in food and frequency of medication intake. On the other hand, it has been shown that Ramadan fasting can be considered as an ideal hypo-caloric diet and a good opportunity to lose weight for patients with type 2 diabetes, particularly for those who are obese or overweight.

Many studies and literature reviews on Ramadan reveal the controversial results concerning the effects of
Ramadan fasting among type 2 diabetic patients, according to anthropometric parameters and/or nutritional intake ${ }^{[1,5]}$. They show an increase ${ }^{[4,6,9]}$, a decrease $\mathrm{e}^{[5,10,11]}$ or no significant change in body weight $^{[1,12,14]}$. Most of these authors suggest that the decrease in weight is related to a decrease in calorie intake, while the increase is correlated with a food excess and reduction of daily activities.

Concerning diet during Ramadan, people usually eat 2 meals, one before dawn, Sahur, and one just after sunset, Iftar. Few studies have assessed the food intake for type 2 diabetics and those deliver conflicting results. A decrease in daily calorie intake has been seen as one of the Ramadan fasting advantages ${ }^{[5,14,15]}$.

Regarding the effect of fasting during Ramadan on the diabetic lipid profile, few studies have been performed and the recommended percentage of calories from fat is dependent on identified lipid problems and treatment goals. Some authors have reported that fasting doesn't affect TGs, LDL-c and HDL-c

Corresponding Author: Méghit Boumédiène Khaled, Department of Biology, Faculty of Sciences, Djillali Liabes University, Sidi-bel-Abbes, Algeria Tel: (+213) 551152261 
rates $^{[4,15,18]}$. However, a decrease in TGs has been noticed $^{[4,8,14,16,18]}$, when TC ${ }^{[5,10]}$, HDL-c ${ }^{[9,13,14]}$ and LDL$\mathrm{c}^{[5,10,15,19]}$ rose. A decrease of HDL-c rate was seldom observed $^{[5,19] \text {. }}$

The present study investigated the effect of a Ramadan fasting diet on the lipid profile of 89 obese women with type 2 diabetes living in Sidi-bel-Abbes city.

\section{MATERIALS AND METHODS}

Patients and study design: The study was undertaken during Ramadan of 2005 (Islamic year 1426), in the Petit-Vichy Diabetes Centre and Hospital University of Sidi-Bel-Abbes city.

One hundred and two patients were recruited, 8 were excluded because of their menstruation, 5 withdrew, and the remainder completed the study. The 89 volunteer out-patients consisted of type 2 diabetic obese women (BMI>30), aged 52 ( \pm 5 years), whose diabetes was identified 4.7 ( \pm 2.6 years) ago and presented no degenerative complications. We focused our choice on diabetic and obese people since prevalence of diabetes is primarily attributable to the rising rate of overweight and obesity ${ }^{[20]}$. Subjects were chosen if they had had diabetes for less than 5 years, because those who were preselected with over 3 years of diabetes presented some degenerative complications and were excluded. In addition women were chosen for this study with the knowledge that another study would be done with male subjects. Throughout the study, diabetics were receiving oral treatment, metformin: Glucophage ${ }^{\circ} 850 \mathrm{mg}$, alone as monotherapy or associated with glimepiride: Amarel ${ }^{\circledR} 3$ or $4 \mathrm{mg}$ as bitherapy. During fasting days, 29 patients were on single hypoglycaemic therapy (metformin) twice daily, the first one was taken at dawn and the second one at sunset (when fast was broken). The other 60 patients on bitherapy (metformin and glimepiride) once daily before the first meal and with the same dose, i.e. metformin $850 \mathrm{mg}$, glimepiride 3 to $4 \mathrm{mg}$. None of the diabetics, who gave their consent following the explanation of the study protocol of the study according to our ethic committee, followed any special restricted diet, but some guidance on how to manage medication during Ramadan was given. Finally, women who were pregnant or breastfeeding, and/or under contraceptives, were excluded from the study.

Methods: The study lasted three months and was scheduled over three periods: one week before Ramadan (T1: pre-fasting), during the month of Ramadan (T2: fasting at the 21st day), and one month after Ramadan (T3: post-fasting). The protocol of the study included a questionnaire in the form of an individual face-to-face interview. The objective of the interview was to gather socio-demographic data, current treatment, body weight status, lifestyle change during Ramadan and frequency of hypo or hyperglycaemia. Three measurements were taken and then averaged for weight and height. The body weight was measured with an electronic balance (SECA®Germany, SECA 731 Sauna: Capacity: $150 \mathrm{Kg}$ Graduations $^{-1}$ : $\left.1000 \mathrm{~g}\right)$. The body mass index (BMI) was defined as follow: BMI $\left(\mathrm{kg} \mathrm{m}^{-2}\right)=$ weight $(\mathrm{kg})$ height $^{-2}\left(\mathrm{~m}^{2}\right)$. Waist to hip ratio (WHR) was determined by measuring the waist circumference (WC) at the narrowest part of the torso and the hip circumference in a horizontal plane at the level of the maximal extension of the buttocks. All anthropometric parameters (weight, BMI, waist and hip circumferences) were noted throughout the three periods (T1, T2, and T3).

The nutrient intake was evaluated by means of a 3 days food record. To this end, all of the patients were instructed on how to record their daily dietary intake. Food quantities were calculated using household measurements (plate, glass, slice, bowl, spoon, etc.) during the three periods. Then individual records were reviewed, and the nutrient calculations were carried out using the USDA's food composition table ${ }^{[21] .}$ Energy intake and diet composition nutrients were calculated with the Nutrinux v1.0 (C) software. Concerning the metabolic control, blood samples were drawn after $12 \mathrm{~h}$ of overnight fasting and were collected over the 3 periods. The measure of FSG, glycated hemoglobin (HbA1c), and lipid components (TC, TGs, HDL-c, LDL-c, apoA-I, and apoB) presented the biological evaluation for the present study.

The FSG, TC, and TGs concentrations were measured by enzymatic methods using the Opera Bayer (Bayer, USA) auto-analyzer and an ion exchange resin separation served to determine the value of HbA1c. The Human Cholesterol Liquicolor test kit (Human, Germany) was used to measure HDL-c, while the LDLc concentration was determined using the Friedewald formula ${ }^{[22]}$. The serum concentration of apoA-I and apoB was determined by a turbidimetric test using a specific antibody (Spinreact, Spain). The anti apoA-I, or anti apoB antibodies form insoluble complexes when mixed with samples containing apoA-I or apoB. These complexes cause a change in absorbance that is dependent on the apoA-I or apoB concentration of the patient sample. They were compared to a pooled reference serum in which the concentration of each was known. 
Statistical analysis: Values are expressed as means \pm SD. Analyses were performed using the Statistical Package for the Social Sciences (SPSS) version 15.0 (Chicago, IL, USA). Group means were compared using Student's paired t-test and one-way ANOVA. Linear regression analysis was performed for determining the relationship between SFA consumption during Ramadan and the rates of TC and LDL-c. A Pvalue of less than 0.05 was considered as statistically significant.

\section{RESULTS AND DISCUSSION}

About 89 women with type 2 diabetes were selected for the study. The physical characteristics are summarized in Table 1.

\section{Anthropometric parameters}

The main finding of this study was a significant decrease in body weight during $\mathrm{T} 2$, in diabetic group $(-2.96 \mathrm{~kg}, \mathrm{p}<0.01)$. In diabetics, the loss of weight was not maintained, and they regained about $1.66 \mathrm{~kg}$ one month later. The circumference measurements i.e. WC and WHR, showed no significant difference during the three periods (Table 1).

Biological parameters: The different rates of blood component analyzed over the 3 periods are indicated in Table 2. In the diabetic group, Ramadan fasting induces a significant decrease in FSG $(-18.84 \%, \mathrm{p}<0.01)$. A decrease in HbA1c $(-7.72 \%)$ but not statistically significant, which was maintained one month after Ramadan period (T3) particularly for HbA1c values.
Ramadan fasting entails major change for the lipid components. A significant increase was found for TC in diabetic group: $+30.77 \%, \mathrm{p}<0.001$. These values fell during T3. Diabetic women showed a significant increase in serum concentrations of TGs $\left(2.47 \pm 0.65 \mathrm{mmol} \mathrm{L}^{-1}\right)$, LDL-c $\left(3.12 \pm 0.50 \mathrm{mmol} \mathrm{L}^{-1}\right)$, and apoB $\left(1.29 \pm 0.36 \mathrm{mmol} \mathrm{L}^{-1}\right)$. This increase was associated with a decrease of body weight, particularly for TC rate that was significantly correlated with weight loss in diabetics $\left(\mathrm{r}^{2}=-0.274, \mathrm{p}<0.05\right)$. As you will see in Table 1, no difference was seen between T1 and T3 serum concentrations of TC and triglycerides (TGs). In diabetic women, apoA-I level was lower during fasting days (T2) than $\mathrm{T} 1$ and decreased by $16 \%$. Through not significantly, HDL-c rate decreased during Ramadan.

Concerning coronary heart disease (CHD) risk indicators such as apoB/apoA-I, TC/HDL-c ratios, results showed high values during all three periods, particularly during Ramadan when a significant increase was observed.

Food consumption: In diabetic women, the meal frequency decreased during $\mathrm{T} 2 \quad(2.1 \pm 0.4)$ when compared to $\mathrm{T} 1(4.4 \pm 0.3)$ and $\mathrm{T} 3(4.1 \pm 0.3)$, and contributed to decreasing the total energy intake (TEI) (data not shown). However, in spite of this decrease the dietary fat consumption, particularly the saturated one, increased significantly $(\mathrm{p}<0.001)$ and represented a high amount (42.14\%) of total fat calories (i.e. $16.05 \%$ of the TEI). During Ramadan not only more fatty foods, but also more dietary cholesterol $\left(377 \pm 131 \mathrm{mg} \mathrm{d}^{-1}\right)$, found their way into the subject's diet.

Table 1: Anthropometric and biochemical parameters during the 3 periods of evaluation

\begin{tabular}{|c|c|c|c|c|c|c|c|c|}
\hline \multirow[b]{2}{*}{ Blood components } & \multicolumn{7}{|c|}{ Diabetic women $(N=89)$} & \multirow[b]{2}{*}{ Recom*** } \\
\hline & $\mathrm{T} 1$ & $\mathrm{~T} 2$ & T3 & $\begin{array}{l}\text { Mean difference } \\
\text { T1/T2 }\end{array}$ & $\begin{array}{l}\text { Mean } \\
\text { standard error }\end{array}$ & & p-value** & \\
\hline Weight $(\mathrm{kg})^{*}$ & $83.56 \pm 9.41$ & $81.00 \pm 9.42$ & $82.64 \pm 9.63$ & $2.6000 \pm 1.700$ & 0.20000 & 14.50 & 0.000 & - \\
\hline $\operatorname{BMI}\left(\mathrm{kg} \mathrm{m}^{-2}\right)$ & $34.67 \pm 2.87$ & $33.39 \pm 2.44$ & $34.11 \pm 3.04$ & $0.5000 \pm 0.900$ & 0.10000 & 5.00 & 0.000 & - \\
\hline $\mathrm{WC}(\mathrm{cm})$ & $106.50 \pm 6.80$ & $106.40 \pm 6.90$ & $106.60 \pm 6.80$ & $2.6000 \pm 1.700$ & 0.20000 & 14.45 & 0.001 & - \\
\hline WHR & $0.88 \pm 0.06$ & $0.88 \pm 0.05$ & $0.88 \pm 0.05$ & $-0.0003 \pm 0.002$ & 0.00024 & -1.07 & 0.287 & - \\
\hline FSG $\left(\mathrm{mmol} \mathrm{L}^{-1}\right)^{*}$ & $9.23 \pm 1.08$ & $8.23 \pm 0.75$ & $8.31 \pm 0.93$ & $1.0100 \pm 1.100$ & 0.12000 & 8.66 & 0.000 & $5.0-7.2$ \\
\hline $\mathrm{HbA1c}(\%)$ & $8.91 \pm 1.06$ & - & $7.96 \pm 0.70$ & $0.9400 \pm 0.950$ & 0.10000 & 9.33 & 0.000 & $<7$ \\
\hline $\mathrm{TC}\left(\mathrm{mmol} \mathrm{L}^{-1}\right)$ & $6.10 \pm 0.81$ & $7.51 \pm 1.55$ & $6.89 \pm 0.94$ & $-1.4200 \pm 1.650$ & 0.18000 & -8.11 & 0.000 & $<5.18$ \\
\hline HDL-c $\left(\mathrm{mmol} \mathrm{L}^{-1}\right)$ & $0.98 \pm 0.21$ & $0.92 \pm 0.22$ & $0.93 \pm 0.21$ & $0.0400 \pm 0.160$ & 0.02000 & 2.83 & 0.006 & $>1.1$ \\
\hline LDL-c $\left(\mathrm{mmol} \mathrm{L}^{-1}\right)$ & $2.42 \pm 0.82$ & $3.41 \pm 0.90$ & $2.68 \pm 0.87$ & $-0.9900 \pm 0.980$ & 0.10000 & -9.50 & 0.000 & $<2.6$ \\
\hline TGs $\left(\mathrm{mmol} \mathrm{L}^{-1}\right)$ & $1.66 \pm 0.63$ & $1.92 \pm 0.71$ & $1.76 \pm 0.65$ & $-0.2500 \pm 0.700$ & 0.07000 & -3.32 & 0.001 & $<1.7$ \\
\hline apoA-I $\left(\mathrm{mmol} \mathrm{L}^{-1}\right)$ & $0.75 \pm 0.20$ & $0.58 \pm 0.18$ & $0.56 \pm 0.19$ & $0.1700 \pm 0.190$ & 0.02000 & 8.43 & 0.000 & $>1.0$ \\
\hline apoB $\left(\mathrm{mmol} \mathrm{L}^{-1}\right)$ & $1.01 \pm 0.21$ & $1.63 \pm 0.23$ & $1.31 \pm 0.21$ & $-0.6200 \pm 0.160$ & 0.02000 & -36.71 & 0.000 & $<1.30$ \\
\hline apoB/apoA-I & $1.39 \pm 0.26$ & $3.02 \pm 0.78$ & $2.24 \pm 0.34$ & $-1.6300 \pm 0.800$ & 0.09000 & -19.34 & 0.000 & 1.75 \\
\hline TC/HDL-c & $6.51 \pm 1.56$ & $8.93 \pm 5.22$ & $7.41 \pm 4.48$ & $-2.4300 \pm 5.070$ & 0.54000 & -4.52 & 0.000 & 6.69 \\
\hline
\end{tabular}

*: Data are given as mean \pm Standard deviation. T1, T2, and T3: indicate before, during, and after Ramadan fasting period respectively. BMI: Body mass index, WC: Waist circumference, WHR: Waist-to-hip ratio, FSG: Fasting serum glucose, HbA1c: Glycated haemoglobin, TC: Total cholesterol, HDL-c: High density lipoprotein cholesterol, LDL-c: Low density lipoprotein cholesterol, TGs Triglycerides, apoA-I: apolipoprotein A1, apoB: apolipoprotein B. **: Compared to pre-fasting period (T1), ***: Recommendation of ${ }^{[23,24]}$ 
It is usually accepted that obesity increases the risk of cardiovascular complications and insulin resistance, and consequently complicates management among type 2 diabetics. There are three important goals for overweight and obese people, particularly for type 2 diabetics, are: a moderate weight loss of about $5 \%$, a lifestyle modification with reduction of energy intake, and an increase in physical activity ${ }^{[23]}$. These factors improve glycaemia and blood pressure control, improve insulin action, decrease fasting blood glucose concentrations, reduce the need for diabetes medications, as well as improve serum lipid concentrations ${ }^{[20,25]}$. It has been noticed that during Ramadan fasting, overweight subjects lose more weight than normal or underweight people ${ }^{[26]}$. This finding is in accordance with our research, results which show a significant $(\mathrm{p}<0.01)$ weight loss $3.12 \mathrm{~kg}$ (i.e. $3.70 \%$ of body weight) during the fasting period. The decrease in meal frequency $\left(\mathrm{r}^{2}=0.323, \mathrm{p}<0.01\right)$ contributed to loss of weight and consequently to the decrease in calorie intake $\left(\mathrm{r}^{2}=0.417, \mathrm{p}<0.001\right)$ during T2. T3 was characterized by a significant $(\mathrm{p}<0.01)$ increase in weight about $2.45 \mathrm{~kg}$, which was apparently due to an increase in energy intake $\left(r^{2}=0.384, p<0.001\right)$. Our results are in line with those of ${ }^{[4,26]}$. The other anthropometric parameters (WC, WHR) showed no significant change.

Concerning diet during the holy month of Ramadan, results reported a significant decrease in the amount of carbohydrates in diabetic women (data not shown) who tried, following their physician's advice, to abstain from eating sweet or starchy foods, in order to reduce calorie intake and improve their glucose control. Consequently, to attenuate their hunger, they consumed more fatty foods.

Improvement of glucose homeostasis during Ramadan was marked by a significant decrease in FSG and HbAlc. The decrease in HbAlc level was correlated with the decrease in TEI $\left(\mathrm{r}^{2}=0.411\right.$, $\mathrm{p}<0.001)$ and was maintained 3 weeks after Ramadan. Nomani et al. ${ }^{[27]}$ suggest that the FSG and $\mathrm{HbAlc}$ values depend on diet composition, energy metabolism, and energy intake regulation. It has been proven that for every $1 \%$ decrease in $\mathrm{HbAlc}$, there is a $35 \%$ decrease in the risk of micro vascular complication ${ }^{[23]}$.

Nomani noticed that the quality of the diet during Ramadan governed the serum cholesterol level ${ }^{[28]}$ and tremendously affected the lipid profile ${ }^{[29] .}$ More dishes based on fat and proteins (i.e. eggs, meat, poultry, salads with mayonnaise, fried foods, etc.) were still consumed during this period. In diabetic women, we recorded a significant increase in TC, TGs, and LDL-c rates, correlated with the amount and with the quality of

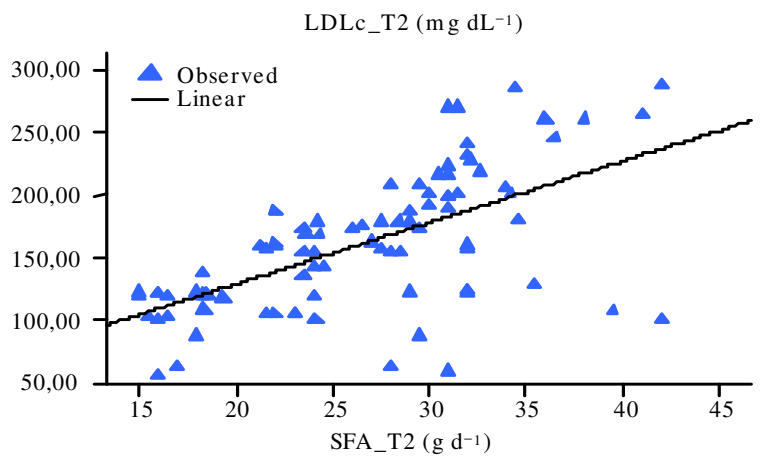

(A)

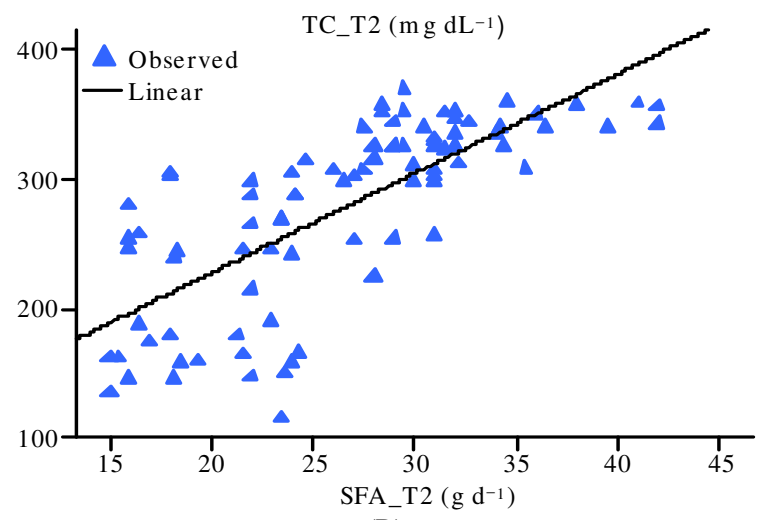

(B)

Fig. 1: The Relationship between LDL-c (A), TC (B) and the amount of SFA brought by Ramadan fasting diet in diabetic women. SFA_T2: Saturated fatty acids during Ramadan, TC/LDL-c_T2: Total cholesterol and LDL-c concentration during Ramadan

dietary fat, particularly saturated ones ( $>15 \%$ of TEI) and dietary cholesterol $\left(397 \pm 124 \mathrm{mg} \mathrm{d}^{-1}\right)$, as shown in Fig. 1. When compared with healthy people, type 2 diabetics present a higher cholesterol synthesis ${ }^{[30]}$. According to Nomani's suggestion ${ }^{[28]}$, dietary fat increases TEI more than $30 \%$ when calorie intake is limited. Our results are in agreement with those of Bougerra et al. ${ }^{[31]}$ and Akanji et al. ${ }^{[32]}$, in which apoB, apoB/apoA-I and TC/HDL-c ratios increased during Ramadan fasting. Type 2 diabetes, as a component of the metabolic syndrome, is commonly accompanied by other CVD risk factors, such as dyslipidemia, hypertension, obesity, and prothrombotic factors. For example, patients with type 2 diabetes frequently have hypertriglyceridemia together with hyper-apoB, an atherogenic lipid profile that often remains unidentified because of concomitant low or normal levels of LDL$\mathrm{c}^{[33]}$. Literature shows that it would be better to measure 
apoB, which is directly involved in a pathologic process, like atheroma, rather than a surrogate, such as cholesterol, and more easily identifiable than LDL-c ${ }^{[34]}$. However, the apoB/A-I ratio is the most important risk factor and cannot be improved by adding any other lipid parameter or ratio, because diabetics present substantial risk with seemingly normal LDL-c rate with high apoB values ${ }^{[35,36]}$. Furthermore, patients with small, dense LDL-c will also typically have lower HDL-c and elevated TGs blood levels, which may increase arteriosclerosis risk.

Fasting from dawn to sunset could cause problems with drug dosage regimens for type 2 diabetics. According to Shaik et al. ${ }^{[37]}$, patients taking metformin, like diabetics with obesity, are at no risk of hypoglycaemia. They suggest that pre-Ramadan doses should be reversed, so that the morning dose is taken after sunset and the evening dose taken with the predawn meal. Zargar et al. ${ }^{[38]}$ suggested that the modification of lifestyle and food intake, which occur during this period, should take into account an appropriate oral agent. No frequent hypoglycaemia episodes were observed in our own patients treated with metformin and/or glimepiride medication. Al-Arouj et $a l .{ }^{[39]}$ reported in their article that patients treated with metformin alone may safely fast because the possibility of hypoglycemia was minimal, and recommended that two thirds of the total daily dose be administered immediately before the sunset meal, while the other third be given before the predawn meal. However, glimepiride with its longer action may offer an advantage for diabetic fasters as reported by ${ }^{[40,41]}$, and didn't make a significant change in glucose and lipid parameters according to a recent Turkish study ${ }^{[42]}$. During Ramadan some of our subjects arbitrarily reduced their drug dosage or even stopped completely and developed hypoglycemia as a result. It should be noted that during the fast, no signs or symptoms of hypoglycemia were observed in any of the patients, except for three cases, two patients had taken their medication without eating the Sahur meal and one exerted an important amount of physical activity effort. In general, fasting during Ramadan seemed to be suitable for almost all diabetic patients and constituted an opportunity to lose some kilograms as well as reduce calorie intake.

\section{CONCLUSION}

It is often said that the beneficial effects of fasting during Ramadan will only occur in patients who maintain their appropriate diets ${ }^{[43]}$. During Ramadan, many diabetics fast without medical guidance and more often than not end up developing acute complications and metabolic disorders, such as hypoglycaemia and blood lipid imbalance ${ }^{[44,45]}$. Because of the effect of obesity on the management of diabetes, weight loss is an important therapeutic objective for obese people who are type 2 diabetics. Ramadan fasting represented a good opportunity for the patients we studied to lose weight and indeed induced a significant weight loss. We attribute this improvement to the decrease in the number of meals. Unfortunately, this loss of weight in diabetics was not maintained during the month following Ramadan, because they fell back into their old eating habits and lifestyles.

The main finding was that FSG and HbAlc value improvement during Ramadan correlated with the decrease in TEI and was successfully maintained 3 weeks after the Ramadan month. This study was exclusively highlighted by the key role played by the diet on lipid profile and the considerable impact of the quality of dietary fatty acids consumed during Ramadan on serum lipid components. The principal factor consisted in SFA, which contributed to increase the LDL-c and TC concentrations.

Education by physicians and nutritionists appears to have a major effect on diabetes management during Ramadan. This is related to a better understanding of the patho-physiology of diabetes during fasting and therefore leads to a greater commitment to self-care.

\section{ACKNOWLEDGEMENT}

Authors gratefully acknowledges Alicia Cleaver who provided assistance in the revision of this manuscript, and thanks all the Laboratory staff of SidiBel-Abbès Hassani Abdelkader University Hospital for technical assistance, particularly the Petit-Vichy Diabetes Centre of Sidi Bel-Abbes. Financial support from Toxicology Research Laboratory of Djilalli Liabes University is thankfully acknowledged too.

\section{REFERENCES}

1. Sulimani, R.A., M. Laajam and O. Al-Attas et al., 1991. The effect of Ramadan fasting on diabetes control type 2 diabetic patients . Nutr. Res., 11: 261-264.

2. Laajam, M.A., 1990. Ramadan fasting and non insulin-dependent diabetes: effect of metabolic control. East. Afr. Med. J., 67: 732-736.

3. Benaji, B., N. Mounib and R. Roky et al., 2006. Diabetes and Ramadan: Review of the literature. Diab. Res. Clin. Pract., 73: 117-125. 
4. Khatib, F. A. and Y.A. Shafagoj, 2004. Metabolic alterations as a result of Ramadan fasting in noninsulin-dependent diabetes mellitus patients in relation to food intake. Saud. Med. J., 25: 1858-1863.

5. Khaled, B.M., M. Bendahmane and S. Belbraouet, 2006. Ramadan fasting induces modifications of certain serum components in obese women with type 2 diabetes. Saud. Med. J., 27: 23-26.

6. Mafauzy, M., W.B., Mohammed and M.Y. Anum et al., 1990. A study of fasting diabetic patients during the month of Ramadan. Med. J. Malayisia, 45: 14-17.

7. Chamakhi, S., B. Ftouhi and N.B. Rahmoune et al., 1991. Influence of the fast of Ramadan on the balance glycemic to diabetics, Medicographia, 13: 27-29.

8. Athar, S. and M. Habib, 1994. Management of stable type 2 diabetes NIDDM during Islamic fasting in Ramadan. In: 1st International Congress on Health and Ramadan, January 19-12, Casablanca, Morocco, pp: 203-212.

9. Khatib, F., 1997. Effect of fasting in Ramadan on blood glucose and plasma lipids in diabetics with NIDDM. In: The 2nd International Congress on Health and Ramadan, December 1-3, Istanbul, Turkey, pp: 42.

10. Yarahmadi, S.h., B. Larijani and M.H. Bastanhagh et al., 2003. Metabolic and clinical effects of Ramadan fasting in patients with type 2 diabetes. J. Coll. Phys. Surg. Pak., 13: 329-332.

11. Klöcker, N., J. Belkhadir and H. El Ghomari et al., 1997. Effects of extreme chrono-biological diet alternations during Ramadan on metabolism in NIDDM with oral treatment. The 2nd International Congress on Health and Ramadan, December 1-3, Istanbul, Turkey, pp: 78-79.

12. Belkhadir, J., H. El Ghomari and N. Klöcker et al., 1993. Muslims with non-insulin dependent diabetes fasting during Ramadan: treatment with glibenclamide, BMJ, 307: 292-295.

13. Uysal, A.R., M.F. Erdogan and G. Sahin et al., 1999. Clinical and metabolic effects of fasting in 41 type 2 diabetic patients during Ramadan. Diabetes Care, 21: 2033-2034.

14. Sari, R., M.K. Balci, S.H. Akbas and B. Avci, 2004. The effects of diet, sulfonylurea and repaglinide therapy on clinical and metabolic parameters in type 2 diabetic patients during Ramadan. Endocr. Res., 30: 169-177.

15. Bouguerra, R., A. Belkadhi and J. Jabrane et al., 2003. Metabolic effects of the month of Ramadan fasting on type 2 diabetes, East. Mediterr. Health J., 9: 1099-1108.
16. Navaei, L., Y. Mehrabi and F. Azizi et al., 2001. Changes in body weight, blood pressure, consumption pattern and biochemical parameters in diabetic patients during fasting in Ramadan. Iran J. Endocrinol. Metab., 3: 125-132.

17. Nagati, K., H. Kamoun and A. Abid et al., 1999. Ramadan Fasting and Diabetes Type II, a Tunisian Multicentric Study. In: The First Congress of Endocrinology and Metabolic Diseases, October 20, 1999, Beirut, Lebanon, pp: 7.

18. Sadr, F., M. Afshar and S. Sarboluki, 2001. Effect of fasting on glucose, lipids, body weight and blood pressure among non-insulindependent diabetes Mellitus. In: The Congress on Health and Ramadan, Tehran, Iran. Iran J Endocrinol Metab., Suppl (Autumn) vol. 18. 2001 (abstract).

19. Jabrane, J., L. Ben Salem and R. Bouguerra et al., 2005. Effect of Ramadan fasting on metabolic balance of type 2 diabetic patient: The 9th Meeting of the Mediterranean Group for the Study of Diabetes April 28-May 1, Nice (France). http://www.mgsd.net/EN/G_espace_menbre/docs/p oster/17.pdf (Accessed in July 2005).

20. Lipscombe, L.L. and J.E. Hux, 2007. Trends in diabetes prevalence, incidence, and mortality in Ontario, Canada 1995-2005: A Population-Based Study. Lancet, 369: 750-756.

21. USDA National Nutrient Database for Standard Reference, http://www.nal.usda.gov/fnic/foodcomp/Data/HG7 2/hg72_2002.pdf

22. Friedewald, W.I., R.I. Levy and D.S. Frederickson, 1972. Estimation of the concentration of low density lipoprotein cholesterol in plasma, without use of the preparative ultracentrifuge. Clin. Chem., 18: 499-502.

23. Abraira, C., W. Duckworth and M. McCarren et al., 2003. Design of the cooperative study on glycemic control and complications in diabetes mellitus type 2 Veterans Affairs Diabetes Trial. Journal of Diabetes and its Complications, 17: 314322.

24. Solano, M.P., and R.B. Goldberg, 2006 Lipid Management in Type 2 Diabetes. Clinical Diabetes, 24: 27-32. .

25. Klein, S., N.F. Sheard and X. Pi-Sunyer et al., 2004. Weight management through lifestyle modification in the prevention and management of type 2 Diabetes: Rationale and strategies. A statement of the american diabetes association, the north american association for the study of obesity and the American Society for Clinical Nutrition. Diabetes Care, 27: 2067-2073. 
26. Azizi, F. and B. Siahkolah, 1998. Ramadan fasting and diabetes mellitus. Int. J. Ramadan Fast. Res., 2: 8-17.

27. Nomani, M.Z., M.H. Hallak, S. Nomani and I.P. Siddiquin, 1989. Changes in blood urea and glucose and their association with energycontaining nutrients in men on hypocaloric diets during Ramadan Fasting. Am. J. Clin. Nutr., 49: 1141-1145.

28. Nomani, M.Z.A., 1997. Dietary fat, blood cholesterol and uric acid levels during Ramadan. Int J. Ramadan Fast. Res., 1: 1-6.

29. Afrasiabi, A., S. Hassanzadeh, R. Sattarivand and S. Mahboob, 2003. Effects of Ramadan fasting on serum lipid profiles on 2 hyperlipidemic groups with or without diet pattern. Saud. Med. J., 24: 23-26.

30. Simonen, P.P., H. Gylling and T.A. Miettinen, 2002. Body weight modulates cholesterol metabolism in non-insulin dependent type 2 diabetics. Obes. Res., 10: 328-335.

31. Bouguerra, R., C.B. Slama and A. Belkadhi et al., 1997. Metabolic control and plasma lipoprotein during Ramadan fasting in non-insulin dependent diabetes. In: Proceeding of the 2nd International Congress on Health and Ramadan, December 1-3, Istanbul, pp: 33 Turkey.

32. Akanji, A.O., O.A. Mojiminiyi and N. Abdella, 2000. Beneficial changes in serum apo A-1 and its ratio to apo $b$ and HDL in stable hyperlipidaemic subjects after Ramadan fasting in Kuwait. Eur. J. Clin. Nutr., 54: 508-513.

33. Sniderman, A.D., T. Scantlebury and K. Cianflone, 2001. Hypertriglyceridemic hyperapob: The unappreciated atherogenic dyslipoproteinemia in type 2 diabetes mellitus. Ann. Int. Med., 135: 447-59.

34. Durrington, P.N., 2002. Can measurement of apolipoprotein $\mathrm{b}$ replace the lipid profile in the follow-up of patients with lipoprotein disorders? Clin. Chem., 48: 401-2.
35. Yusuf, S., S. Hawken and S. Ounpuu et al., 2004. Effect of potentially modifiable risk factors associated with myocardial infarction in 52 countries (the INTERHEART study): Casecontrol study. Lancet, 364: 937-952.

36. Walldius, G. And I. Jungner, 2006. The apo B/apo A-I ratio: A strong, new risk factor for cardiovascular disease and a target for lipidlowering therapy-a review of the evidence. J. Int. Med., 259: 493-519.

37. Shaik, S., D. James, J. Morrissey and V. Patel, 2001. Diabetes care and Ramadan: Fast or no to fast. Br. J. Diabetes Vasc. Dis., 1: 65-57.

38. Zargar, A., A. Basit and H. Mahtab, 2005. Sulphonylureas in the management of type 2 diabetes during the fasting month of Ramadan. J. Indian. Med. Assoc., 103: 444-446.

39. Al-Arouj, M., R. Bouguerra and J. Buse et al., 2005. Recommendations for management of diabetes during Ramadan. Diabetes Care, 28: 2305-2311.

40. Glimepiride in Ramadan (GLIRA) Study Group, 2005. The efficacy and safety of glimepiride in the management of type 2 diabetes in muslim patients during Ramadan (GLIRA). Diabetes Care, 28: 421-422.

41. Anwar, A., K.N. Azmi, B.B. Hamidon and B.A. Khalid, 2006. An open label comparative study of glimepiride versus repaglinide in type 2 diabetes mellitus Muslim subjects during the month of Ramadan. Med. J. Malaysia, 61: 28-35.

42. Cesur, M., D. Corapcioglu and A. Gursoy et al., 2007. A comparison of glycemic effects of glimepiride, repaglinide and insulin glargine in type 2 diabetes mellitus during Ramadan fasting. Diabetes Res. Clin. Pract., 75: 141-147.

43. Rashed A.H., S.A., Siddiqui and S.H. Aburomeh, 1989. Clinical problems during fast of Ramadan. Lancet, 1: 1396 .

44. Qureshi, B., 2002. Diabetes in Ramadan. J. R. Soc. Med., 95: 489-490.

45. Bouguerra, R., C. Jabrane and C. Maâtki et al., 2006. Ramadan fasting in type 2 diabetes mellitus. Ann. Endocrinol., 67: 54-59. 